\title{
Author Correction: ATF4 couples MYC-dependent translational activity to bioenergetic demands during tumour progression
}

Feven Tameire, loannis I. Verginadis, Nektaria Maria Leli, Christine Polte, Crystal S. Conn (D, Rani Ojha,

Carlo Salas Salinas, Frank Chinga, Alexandra. M. Monroy, Weixuan Fu D, Paul Wang (D), Andrew Kossenkov, Jiangbin Ye, Ravi K. Amaravadi, Zoya Ignatova, Serge Y. Fuchs, J. Alan Diehl ID, Davide Ruggero and Constantinos Koumenis (iD

Correction to: Nature Cell Biology https://doi.org/10.1038/s41556-019-0347-9, published online 1 July 2019.

In the version of this article initially published, the first sentence of the final paragraph of the introduction incorrectly stated "... uncharged amino acids." The correct text is "...uncharged tRNAs.... The error has been corrected in the HTML and PDF versions of the article. 